\title{
Dietary interventions and increase of dietary iodine intake - a systematic review
}

\author{
M. Bouga and E. Combet \\ Human Nutrition, School of Medicine, College of Medical Veterinary and Life Sciences, University of Glasgow, \\ Glasgow Royal Infirmary, Glasgow, UK
}

Young UK women are iodine-insufficient at a population level ${ }^{(1)}$. No prophylactic measures address this issue, which impacts on maternal thyroid function and infant neurodevelopment. The UK Department of Health has not reviewed the iodine recommendations for pregnancy and lactation and proposes no increment, in contrast to the WHO/ICCIDD and EFSA which recommend an increase of iodine intake from $150 \mu \mathrm{g} /$ day to $250 \mu \mathrm{g} /$ day and $200 \mu \mathrm{g} /$ day respectively. As controversial salt iodisation is not mandatory in the UK, and as the pregnancy supplements provided by the health services do not contain iodine, pregnant mothers and their infants may be at risk of iodine deficiency disorders. Iodine knowledge and awareness remain low amongst mothers ${ }^{(2)}$ and healthcare professionals. Randomised controlled supplementation trials present ethical difficulties in pregnancy. We propose that simple dietary guidance, such as repositioning dairy and seafood as essential component of the diet during pregnancy and lactation, can address this public health concern.

A systematic review was conducted, in order to identify and analyse the interventions in pregnancy that aimed to increase dietary iodine intake, excluding those that used iodised salt and supplements and those that were not human studies. An extensive search of electronic databases (Web of Science, Pubmed, EMBASE and Epistemonikos) from 1990 to November 2015 was conducted.

A total of 2,501 titles were reviewed by two independent reviewers; 196 publications were considered for abstract screening and 28 for full-text screening. Two studies met the criteria for inclusion in the present review ${ }^{(3,4)}$.

The LIMIT study (South Australia) was an intervention in overweight and obese women, at 10-12 weeks of gestation and followed-up to 4 months postpartum. The intervention group received dietary, physical activity and behavioural strategies, without specific focus on iodine. Iodine intake of the intervention group was modified by maternal BMI category (interaction $\mathrm{P}<0 \cdot 05$ in all cases), with overall intake significantly increased in obese but not overweight women. No significant differences in iodine intake was observed when compared between intervention and control groups $(\mathrm{p}=0 \cdot 38)$.

The second publication was a suggested study protocol of an upcoming cluster randomised controlled multicentre trial in pregnant women in Spain, recruited at the first trimester of pregnancy to their last antenatal appointment of the third trimester. Women in the intervention group would receive group education on healthy hygiene-dietetic habits and the importance of an adequate iodine nutritional status during the first trimester of pregnancy. The consumption of iodine-rich foods and iodine deficiency were described as the outcomes of the study. Findings were not published to this date.

Overall, there is lack of interventional studies that aim to increase the iodine intake of a pregnant population by simple dietary guidance, education, increased awareness or provision/repositioning of iodine rich foods in the diet.

1. Vanderpump MPJ, Lazarus JH, et al. Lancet. 2011;377(9782):2007-2012.

2. Combet E, Bouga M, et al. Br J Nutr. 2015;114(01):108-117.

3. Dodd JM, Cramp C, et al. BMC Med. 2014;12:161.

4. Prieto G, Torres MT, et al. BMC Pregnancy Childbirth. 2011;11(17):17. 